\title{
Probing Documentation Practices: Reflecting on Students' Conceptions, Values, and Experiences with Documentation in Creative Inquiry
}

\author{
Ricky Chen \\ School of Design \\ Carnegie Mellon University \\ rickyche@andrew.cmu.edu
}

\author{
Miso Demko \\ Dietrich College \\ Carnegie Mellon University \\ mdemko@andrew.cmu.edu
}

\author{
Daragh Byrne \\ School of Architecture \\ Carnegie Mellon University \\ daraghb@andrew.cmu.edu
}

\author{
Marti Louw \\ Human-Computer Interaction Institute \\ Carnegie Mellon University \\ mrlouw@andrew.cmu.edu
}

\section{ABSTRACT}

ABSTRACT

This research examines how maker-based teaching and learning impact the ways students practice and value process documentation. Educational environments like design studios and makerspaces rely heavily on the physicality of students' work in hands-on, creative mediums. Transitioning learning and making to remote formats due to the pandemic provided an opportune moment to reflect on creative making curricula and its relationship to space, tools, and materials. Using a research-through-design approach, we deployed a remote design probe to examine students' current understanding, contexts, values, and experiences with documentation and its integration with technology, space, and education. We report our findings from five activities completed over a one-week period by 15 students enrolled in undergraduate interdisciplinary creative programs. We illustrate how students have been navigating documentation practices as they continue to learn and work in new, remote settings. We demonstrate that despite different disciplinary standards, students share common values in how documentation connects with deeper learning processes.

\section{AUTHOR KEYWORDS} Education, Maker Education, Creative Practice

\section{CSS CONCEPTS}

- Human-centered computing Interaction design Empirical studies in interaction design

C\&C '21, June 22-23, 2021, Virtual Event, Italy (c) 2021 Copyright is held by the owner/author(s). ACM ISBN 978-1-4503-8376-9/21/06. https://doi.org/10.1145/3450741.346539

\section{INTRODUCTION}

Documentation tools and practices play a key role in project-based learning by making learners' thinking visible $[7,16,47,48]$ and by revealing process and accomplishments $[7,59]$. This is especially true in creative learning environments such as makerspaces and [1, 9, 44], design, architecture, and art studios [51, 53, 61], where documentation is often a required artefact to evidence learning [10, 40]. Sharing documentation with others is vital to learning as it externalizes concepts and reveals craft $[13,52,53]$, while building community [30, 44]. Yet, the open-ended nature of creative, inquiry-based learning experiences challenges a singular approach to documentation. Through a visual compendium of design process models, Dubberly shows just how varied design as a structured activity can be [19]. Recent work by Gibbons et al. additionally notes that diverse instructional practices, a wide range of learning goals, and disparate values guiding design-based education further complicate the role, practice, and assessment of documentation in learning [25]. As such, in design-, arts-, and maker-based education, evidence of learning is dependent on the instructional goals, the assignments or project intermediaries reviewed, and the learner's own intent, framing, and inquiry process. Further, documentation itself can take multiple forms (from analog to digital), can be some combination of recorded textual, visual, or physical artefacts, and also can be more process or product-oriented. For example, design practices traditionally favor students evidencing learning through design journals to continually reflect on processes, later transitioning to presenting work in an individual portfolio $[6,39]$. More recently, digital documentation on platforms such as Medium, blogs, or online portfolios has become increasingly popular [9] especially for those creative professionals and freelancers involved in "portfolio careers [54]." In engineering and maker-based education, step-by-step guides to reproducing work, such as DIY tutorials, can be a preferred format for disseminating work, building visibility, sharing resources, and engaging broader communities around work products
$[15,56]$. These mechanisms also offer new and alternative formats to support studio culture and for learners to evidence expertise that advances creative hands-on inquiry [31]. For students, it can be challenging to know what and how to document, despite the practice of documentation being a critical competency for evidencing learning, building professional and reflective practices, and connecting to opportunities. While the importance of documentation is often alluded to in design and education literature [50], it is rarely interrogated in practice or compared across disciplinary frames, intents, or approaches, and even more rarely is it studied or discussed from the perspectives of the learners' appreciation for and encounters with documentation in the studio, lab, or makerspace.

To address this gap, we developed an exploratory design probe to better understand how students think about, interpret, and practice documentation in educational settings. We report the insights gathered from this activity, deployed among 15 interdisciplinary undergraduate students at Carnegie Mellon, a private US university, as they navigated the changes and challenges of creative, projectbased inquiry courses and documentation during Fall 2021 and the COVID-19 pandemic. This pictorial offers the following three contributions. First, we discuss the development of this design probe and illustrate how it characterizes student perspectives on this creative learning practice. Second, we demonstrate that the probe not only interrogates documentation, but also affords students a means to coordinate, materialize, and reflect upon a complex set of practices and reveal to themselves the underlying values which motivate their documentation activity. Finally, we discuss how students reconstitute documentation practices when learning at home. This work ultimately offers insights into students' perceptions of documentation and the mediating role it plays in collaborative creative project work in the face of great disruption. 


\section{BACKGROUND}

Studios are materially rich spaces where documentation enacts reflection in real-time through display, conversation, and review around the objects and emergent activities of design, serving as a parallel live workspace $[20,38]$. Within these creative learning environments, the role of documentation is usually cast in two ways-as evidence of learning for assessment or as part of a reflective practice. Despite the importance of documenting work, students' perceptions, values, and practices of documentation in creative fields are understudied.

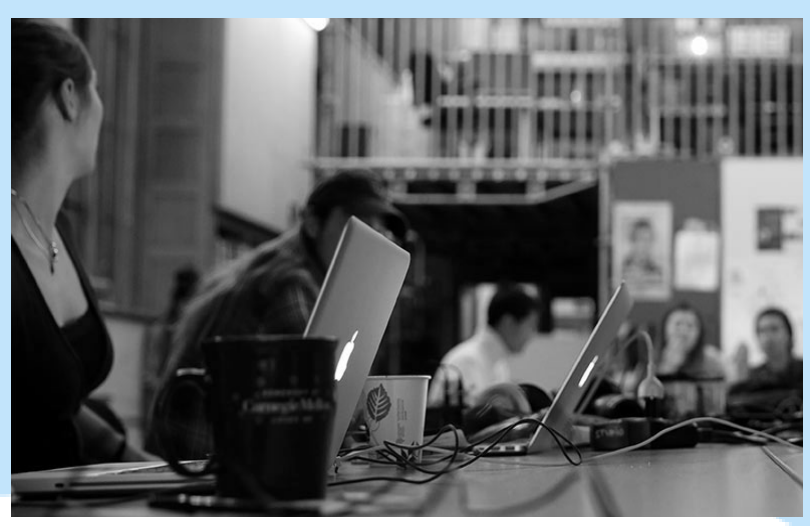

\section{Mediating Technologies}

Student documentation activities are often mediated by computational tools and digital capture devices. Early work in intelligent tutoring by Collins \& Brown [11] introduced a technological innovation that took advantage of computer's inherent capacity to track actions and enabled automatic documentation of students' activity. Subsequent work has built on this notion of automating the capture of digital and tangible constructions in engineering design education $[58,59]$. Other solutions have aimed at using automatically gathered photos to encourage social reflection [21] or structuring understanding of the design process using a visual decision tree representation of documentation artifacts [59]. Despite the feasibility of automating, nudging, and structuring documentation tasks, user motivation, curation effort, and intuitive mechanisms for capture and sharing remain challenging $[9,14]$. Keune et al. suggest this problem can be addressed by asking how documentation can be made more immediate, purposeful, and personally meaningful [44]. They highlight technology's mediating and supporting role in documentation and propose promoting students' own interests, self-initiated processes, and personal adaptation as strategies.

\section{Documentation for Assessment}

Documentation is typically prioritized as an assessment tool for educators. In design and art fields, documentation of project intermediaries and their presentation in reviews and portfolios plays a prominent role in both informal and formal assessments [3, 23, 45]. Documentation is well suited to qualitative assessment of valued skills and competencies as it makes aspects of the creative learning process visible so it can be shared, discussed, reflected upon, and judged [46, 55]. Documentation can be organized into narrated portfolios, design cases, or assembled as a package of assigned learning artefacts or a visual presentation, forms which prioritize formative assessment, interpretive accounts, and evidentiary reasoning $[18,35]$. In addition to supporting assessment, documentation also has student-centered benefits including developing valued domain competencies [51] and connecting to professional opportunities through sharing one's portfolio work [54].

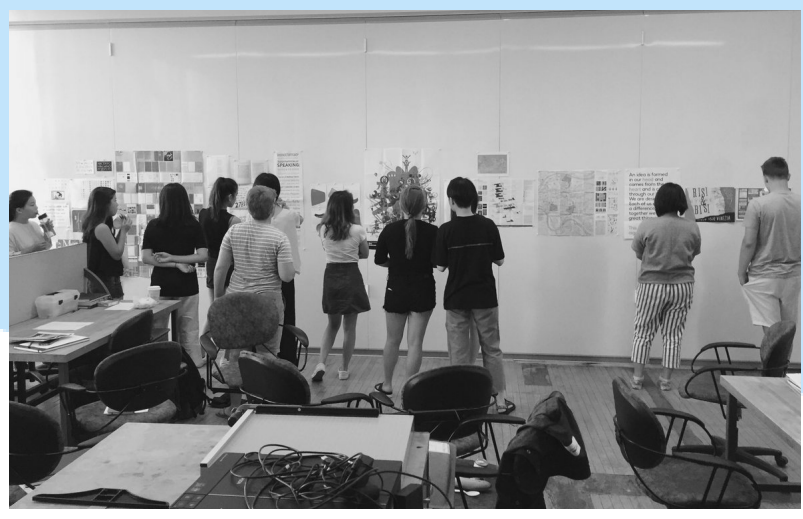

\section{Embodied Reasoning and Participation}

Documentation artefacts support participatory pedagogies where educators and students co-construct meaning in peer and dialogic interactions around works-in-progress [52, 55]. Moreover, embodied reasoning, often through analogy-building gestures, brings documentation artifacts into conversation for collaborative sensemaking and knowledge building [36] during in-person critique sessions. Oak et al. demonstrate how subtle, socially negotiated, intersubjective meanings emerge between individuals in talking about design dilemmas and their resolution [38]. This rich in-person, multimodal process of shared interpretation and reasoning around process artefacts, due to pandemic conditions, moved to a fully online discourse modality for these students this semester.

\section{Documentation for Reflection}

Documentation also plays an important role in learning reflective practice by fostering cyclic internal revisiting of one's process, performance, and progress. [12, 53]. In this way, acts of documentation can serve a metacognitive function in aiding self-regulation routines $[2,28]$ and enabling reflexive moments of self-critique and selfcorrection as learners make their way through a self-directed inquiry process [42]. In digital systems, notions of process and performance are equally important to scaffold reflective practices. Lin et al suggests four mechanisms namely: process displays, process prompts, process models, and forums for reflective social discourse [33]. Alternatively, Fleck \& Fitzpatrick offer a framework that considers the levels, conditions, and techniques that foster reflective behaviors through technology [22]. While documentation is not explicitly evoked in either $[22,33]$, it is the working artefacts that enable reflection to occur that are central to these systems. Tools for documentation often tend to emphasize either the externalized forms for assessment or the latter more contemplative, internalized forms for self-reflection.

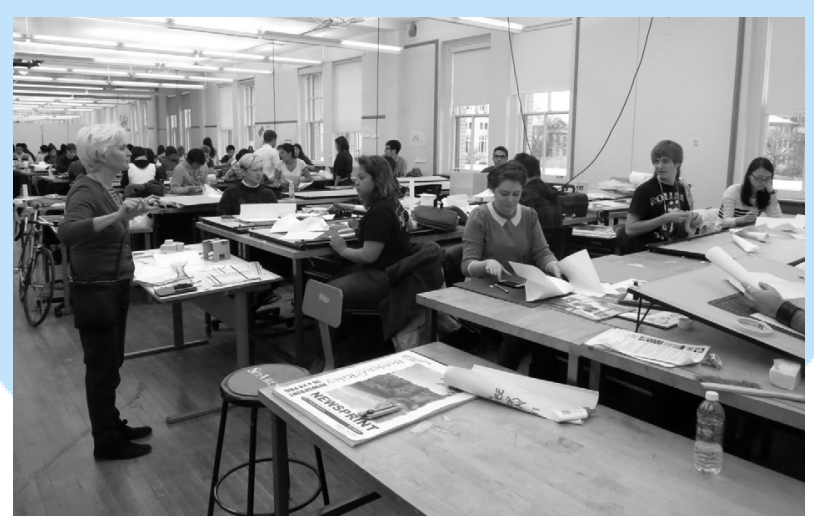

\section{Building Culture and Practice}

Sharing portfolios online improves instruction and fosters learning communities, but implementing consistent portfolio practices in a physical setting confers additional benefits. Formal and informal critique practices around work products are a key feature of studio environments, [55, 61]. Documentation also provides the building blocks for portfolios which enable students to gain peer recognition for their work, model professional practices, and explore the work of others [44]. Given et al. [26] demonstrate that exhibiting - through cycles of documenting, reflecting, and sharing - constitute a deliberate set of routines that made teaching and learning visible and that engages the broader community culture and collective (local) knowledge. Its implementation proved to be culturally specific - something which we also explore in our work. 


\section{BUILDING A PROBE FOR DOCUMENTATION}

The COVID-19 pandemic during the 2020-2021 academic year necessitated that students would be engaged in remote or "hybrid" learning; they would largely be unable to access the typical tools and resources available in makerspaces, nor be able to engage with their peers in the usual ways that support their creative practices. While disorienting, this moment also afforded students an unusual opportunity to notice and reflect on their relationship to documentation in the frictions and gaps that resulted from the abrupt shift to remote learning. As direct observation of classroom activities was infeasible due to pandemic conditions, we opted for a design probe approach as a generative research method well suited to collecting rich, experiential data where in situ observation and other forms of direct inquiry may be challenging. Recognizing documentation's learning benefits along with its pedagogical challenges, we designed our probe to explore an open question:

\section{How do documentation practices support creative learning processes?}

\section{DESIGN PROBES AS A METHOD}

Probes as a loosely defined design research method have been subjected to a variety of interpretations: cultural probes, design probes, technology probes, empathy probes, etc. [24, 27, 34]. Boehner et al. in a comprehensive review of the literature on probes used in HCI called out a set of distinguishing qualities that probes share [4]. Probes are typically designed to be given as a package or kit which usually includes one or more "evocative tasks" to be completed by participants on their own time [49]. Often, probe activities have a provocative, ambiguous, or open-ended quality, and individuals are usually asked to reflect on and record responses to the more "emotional aspects" of the probe experience $[4,27]$. The data collected is intended to provide the design team with a heightened sensitivity and deeper account of the design situation, rather than yielding objectively validated findings. Design probes are often invoked at an early, exploratory stage of a design process to reveal hidden aspects of use, surface tacit behaviors, or uncover new interactions and application possibilities.

\section{BUILDING A SITUATED UNDERSTANDING OF DOCUMENTATION}

\begin{tabular}{|c|c|c|c|c|}
\hline Probe & $\begin{array}{l}1 \\
1\end{array}$ & Focus & $\begin{array}{l}\text { I } \\
\text { I }\end{array}$ & Activity \\
\hline & $T$ & & $T$ & \\
\hline \multirow[t]{2}{*}{1} & 1 & Objects/Tools & I & \multirow[t]{2}{*}{ Annotated Workspace } \\
\hline & I & & I & \\
\hline \multirow[t]{2}{*}{$2 \& 4$} & I & Meaning/Ideas & I & $\begin{array}{l}\text { Concept Maps and } \\
\text { Ranked Lists of Values }\end{array}$ \\
\hline & I & & I & \multirow{3}{*}{$\begin{array}{l}\text { Documentation through Project Workflow and } \\
\text { Highlights (frictions and missed opportunities) }\end{array}$} \\
\hline \multirow[t]{2}{*}{3} & I & Activities & I & \\
\hline & I & & I & \\
\hline
\end{tabular}

\section{SUMMARY OF PROBE ACTIVITIES}

- - - - - - - - - - - - - - - - - - - - - - - - - - - - - - - - - - Our probe packet was built to gain a better understanding of the subjective experience and role of documentation in creative practices. To do this, we decomposed our guiding research question into four more tractable sub-questions, each corresponding to one of the four probe activities.

\section{PROBE 1: Workspace Photo Annotation}

How does the shift from studio-based to remote learning affect documentation setups?
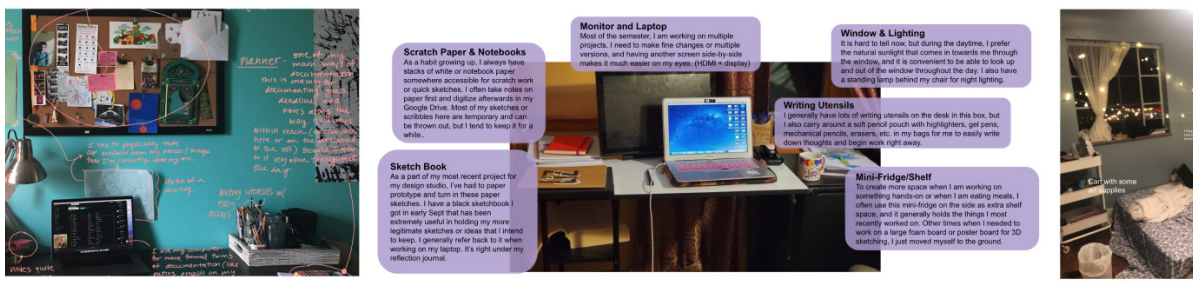

PROBE 2: Mind Mapping

What does "documentation" mean to students, and what conceptions do they associate with the term?
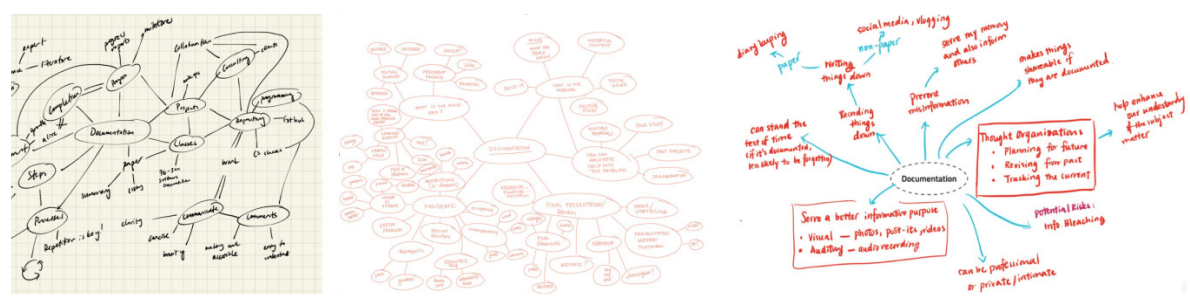

PROBE 3: Project Journey Map

How do students represent their creative processes, and what frictions for documentation are revealed?
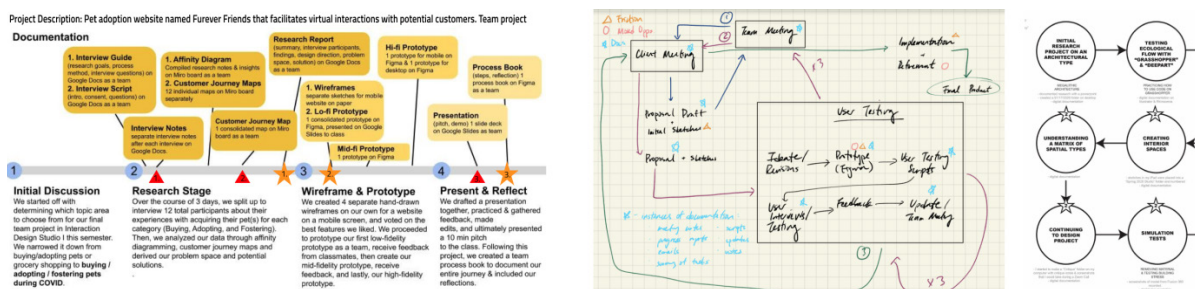

PROBE 4: Listing \& Rating Values

What are the reasons that students practice documentation and how are they prioritized?
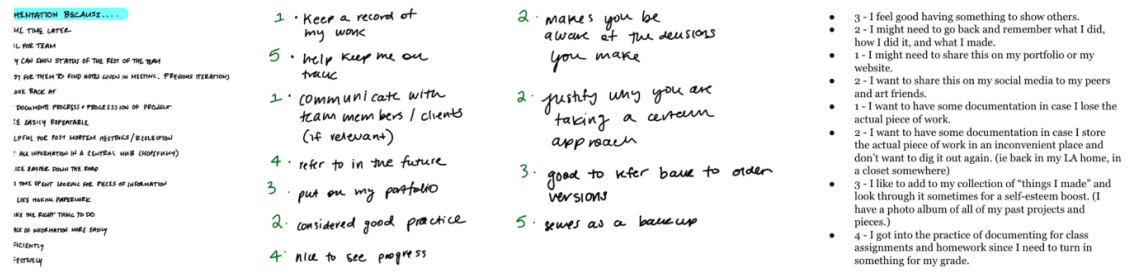


\section{PARTICIPANTS AND STUDY DESIGN}

- - - - - - - - - - - - _- - - - - - - - - - Sixteen undergraduate students (eight seniors, four juniors, four sophomores) were recruited from two interdisciplinary programs at Carnegie Mellon focused on project-based learning and creative inquiry, namely the Human Computer Interaction (HCI) and Integrative Design Arts and Technology (IDeATe) network. Participants were screened to balance for disciplinary backgrounds by majors and minors. One student did not complete the probe. All participants were engaged in remote-learning courses at the time of the study, which was conducted at the end of the Fall 2020 academic semester. All participants had experience with documentation in project-based learning and indicated documentation as being important to their work - half of the participants rated it as extremely important on a 7-point semantic differential scale. Students completed one probe activity each day over four days and were free to use any tools to complete the task. The activities each took approximately 15-20 minutes and on the fifth day, the study concluded with a semistructured debriefing interview with each student. Participants were compensated with a $\$ 50$ gift card.

\section{MOVING FROM DATA TO INSIGHTS}

- - - - - - - - - - - - - - - In responding to the probe activities, each student reflected on the role of documentation in their own learning process and creative practice. Several of the probe activities in themselves represent "creative acts of making [49]." Gathering these artefacts together (see below) allowed the research team to both look across each probe activity to see the range of student responses as well as to look down a column to see a single individual's responses. We segmented participants by major and program to examine how disciplinary backgrounds might influence documentation practices and values. Given the varied nature of elicited materials collected, we used a qualitative artifact analysis technique [43] to inductively code the responses and synthesize insights.

\section{STUDY PARTICIPANT'S ACADEMIC BACKGROUNDS}

ADETMIC BACKGROUNDS

Students' primary majors are listed in the circles corresponding to their participant ID's and each student was either enrolled in $\mathrm{HCl}$, IDeATe or both. The color codes specify the track in IDeATe.

Human-Computer Interaction

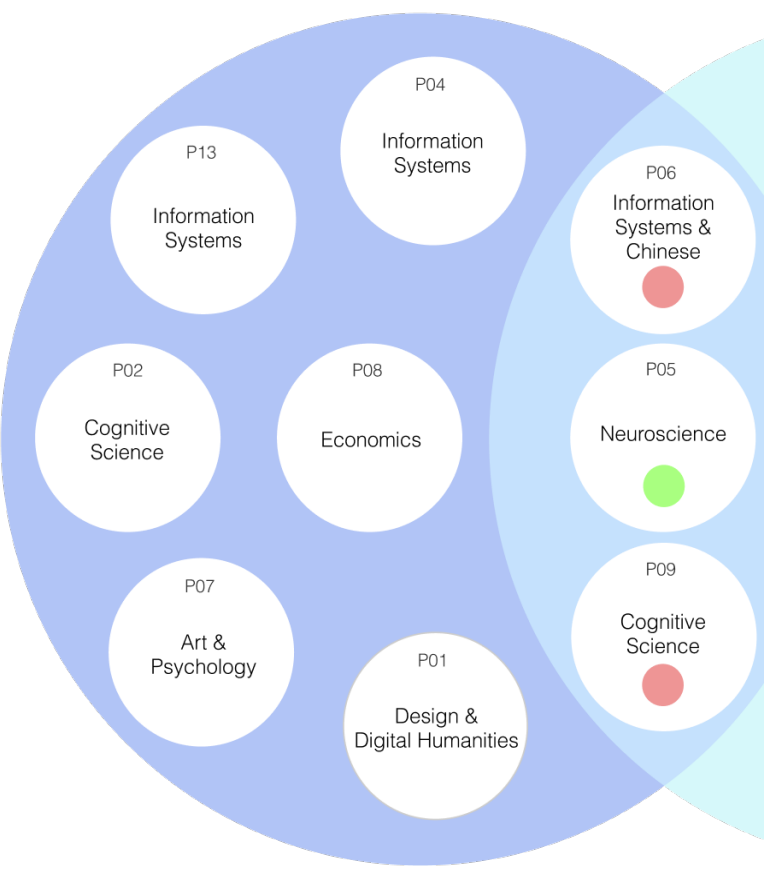

IDeATe

Intormation
Systems

0

Drama Design

(

DeATe Key

Media Design

P03

Business
\& Finance

Architecture

\& Photography

Game Design

Physical Computing

Animation \& Special Effects

Soft Technologies

Architecture

Intelligent Environments

P11

$\mathrm{P} 12$
$\mathrm{x}-\mathrm{C}$

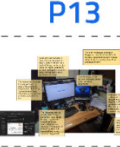

P14
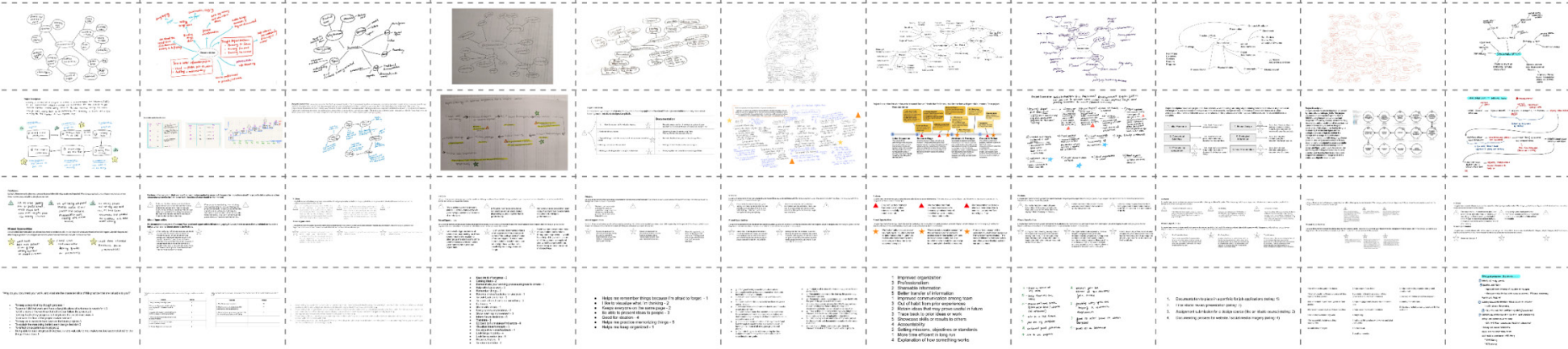


\section{PERSONAL MEANINGS} For one of the activities, participants were asked to complete a mind map in response to the prompt "what does documentation mean to you?" Mind mapping is one of a broad family of graphic organising tools that can include concept mapping [57], argument mapping $[8,17]$, and spider diagramming [60]. Mind maps are also often used in higher education as a reflective tool to reveal and consolidate knowledge [29]. This activity draws on prior research on teaching and learning to surface conceptual models and make word associations visible for analysis [32, 37]. We used this technique to capture how students structured their conceptual understanding of documentation as a domain of knowledge, skills, and practices.

This activity in particular offered a valuable reflective tool for learners themselves to unpack and analyze their own understanding of documentation. Depicted here is a representative subset of the student mind maps. Researcher annotations highlight the varied approaches to conceptualizing documentation, as well as the shared terms and associations found among our participants.

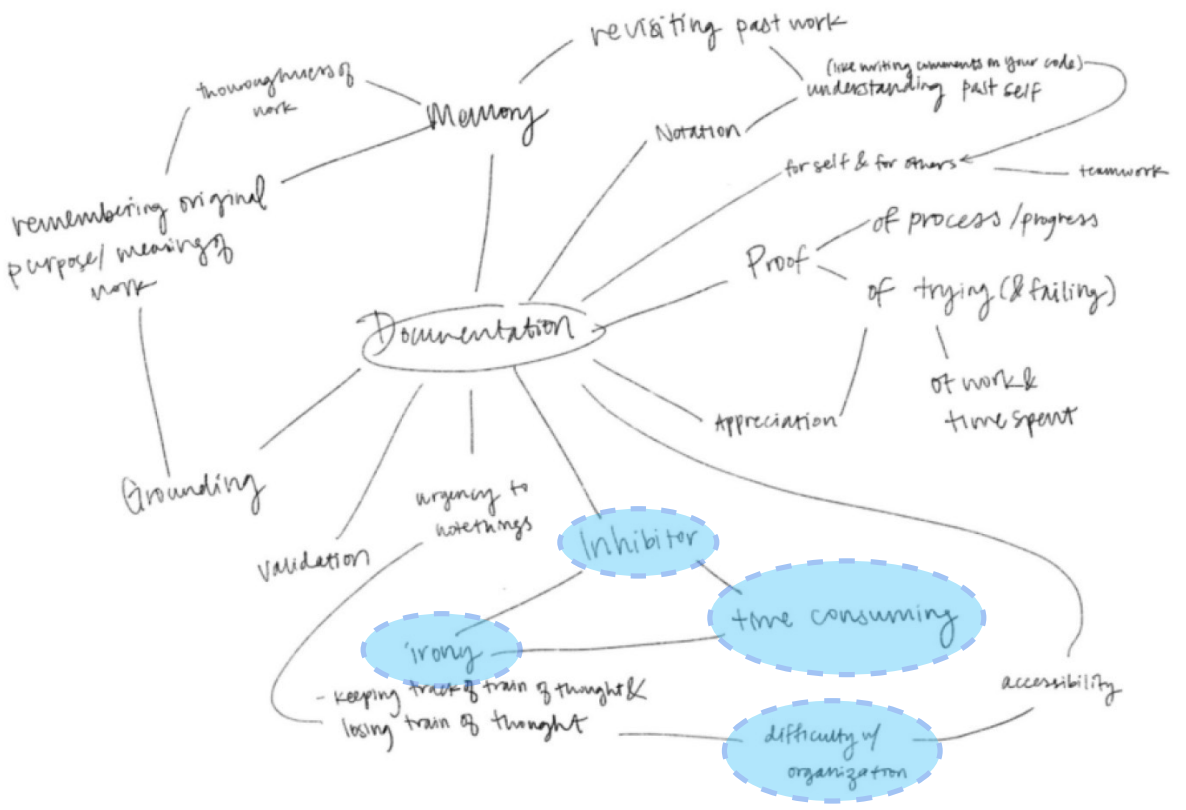

Participant 01 (Design, Digital Humanities, and Human-Computer Interaction)

Participant 01 names several personal benefits to documentation (e.g. aiding memory, selfunderstanding) which are countered by experienced difficulties (e.g. breaking flow, time consuming). Interconnecting lines show fluid relationships between ideas.

$$
\text { makes things }
$$

$$
\text { shaveable if }
$$

$$
\text { they are }
$$

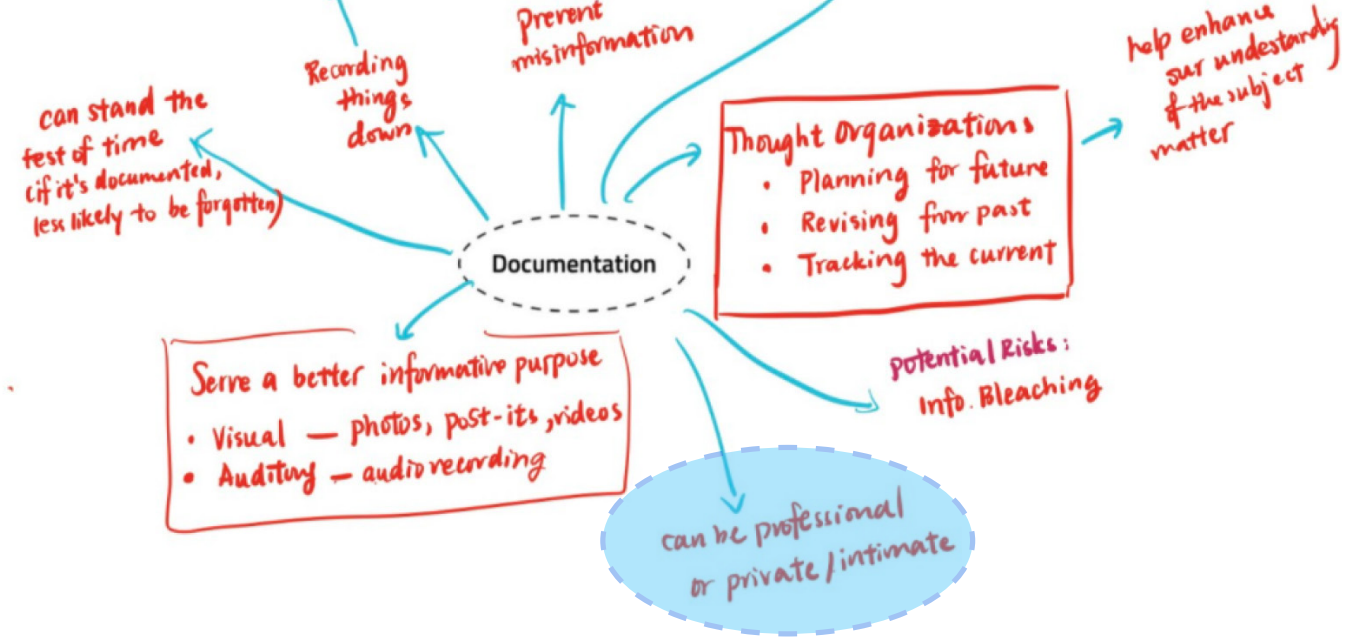

Participant 03 (Business, Finance, and Soft Technologies)

Participant 03 calls out the past (revising), present (tracking), and future (planning) roles documentation plays in organizing thought and notes the role of documentation in preventing misinformation while running the risk of "info bleaching." Other nodes reference memory (informing self and others) and balancing considerations of a professional vs private/intimate divide.
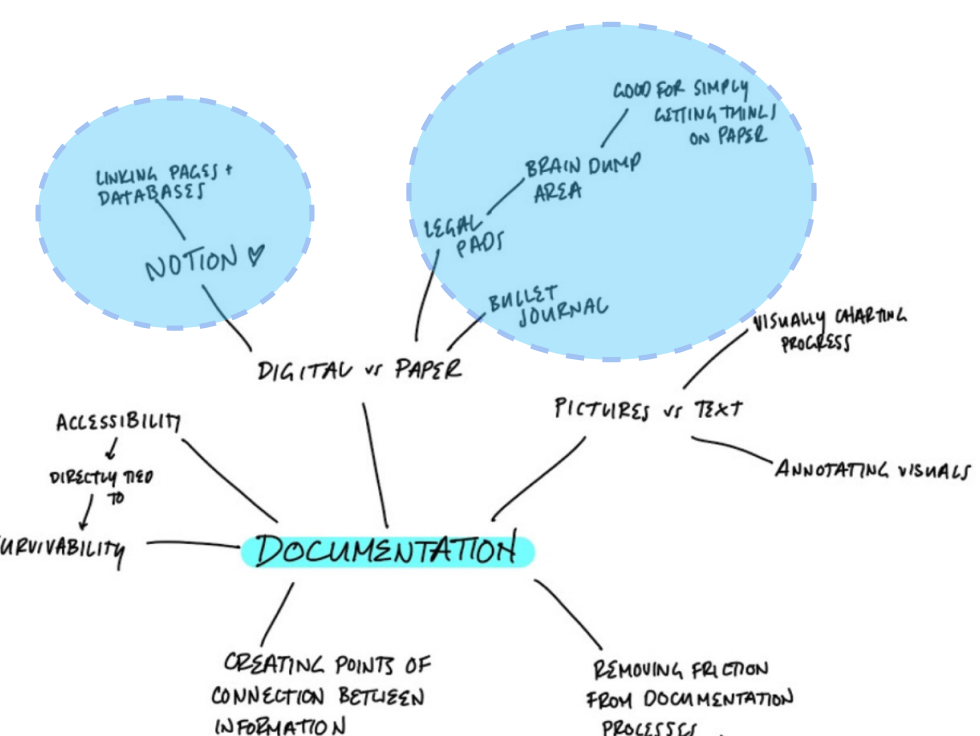

REMOUING FRETTON FROM DOCM MENTATION INFORMATION
Participant 12 also positions documentation amid a set of distinct but connected pairings. They, in particular, attend to the tools that support documentation for them (e.g. journals, Notion), and managing pain points (e.g. removing friction, tediousness). 


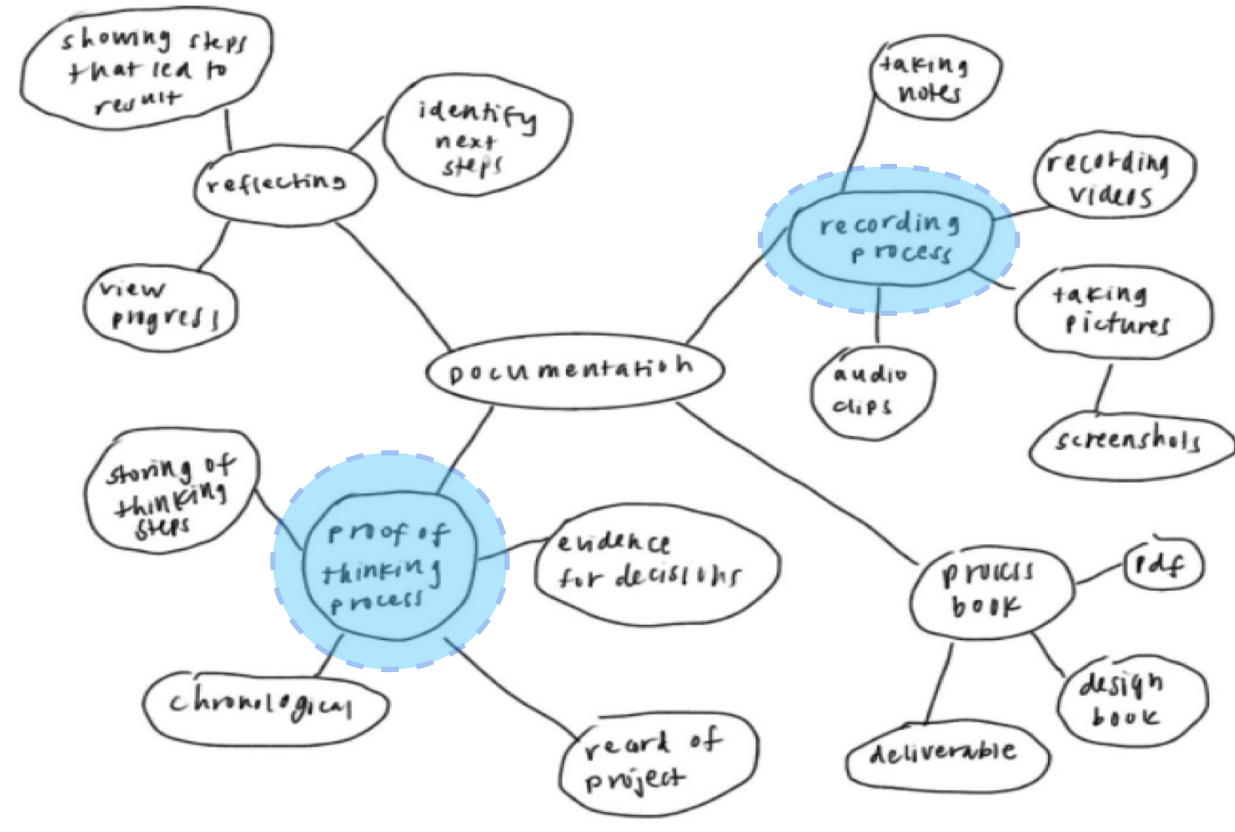

Participant 02 (Cognitive science, Human-Computer Interaction)

Participant 02 recognizes documentation as both consisting of products of recording as well as final/ finalized forms that function as proof or validation. The student demonstrates a "doing" focus.

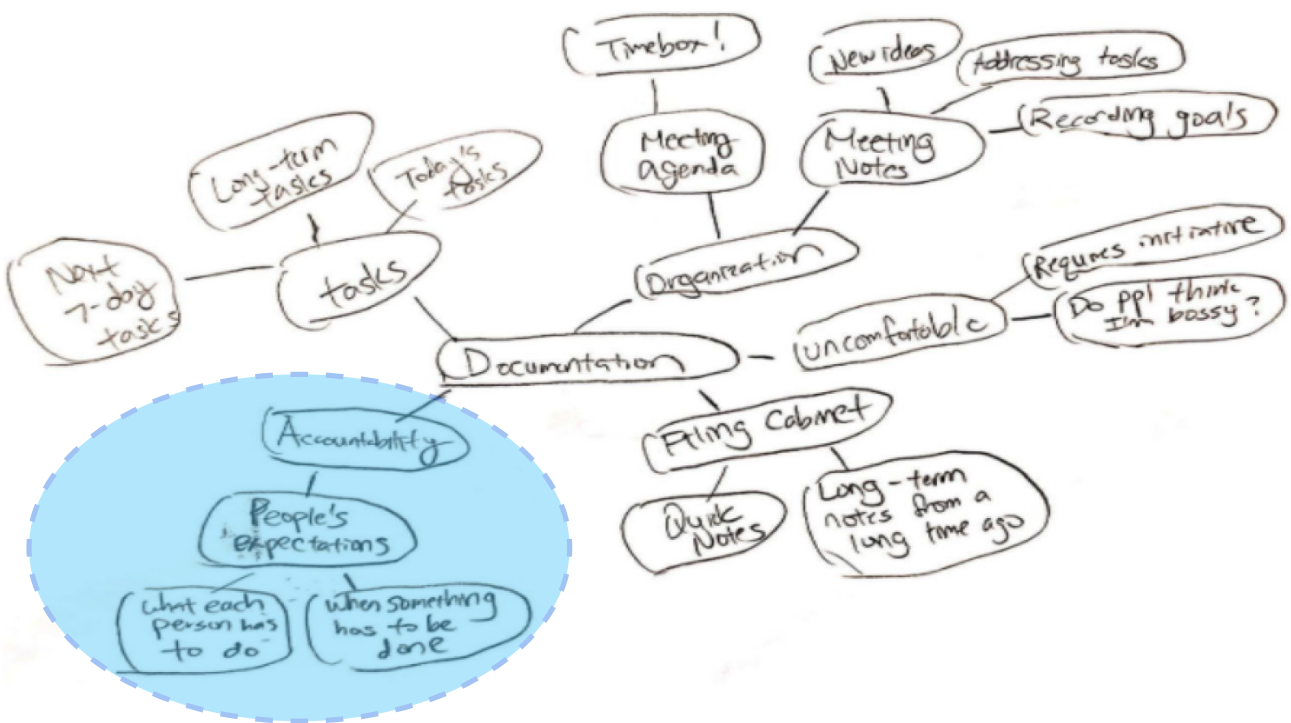

Participant 06 (Information Systems, Chinese, Human-Computer Interaction, Media Design

Participant 06 presents a view of documentation in relation to demands and responsibilities. Pain points are related to group work. Metaphors, like "filing cabinet" and "timebox", are used to explain documentation's role in their process.

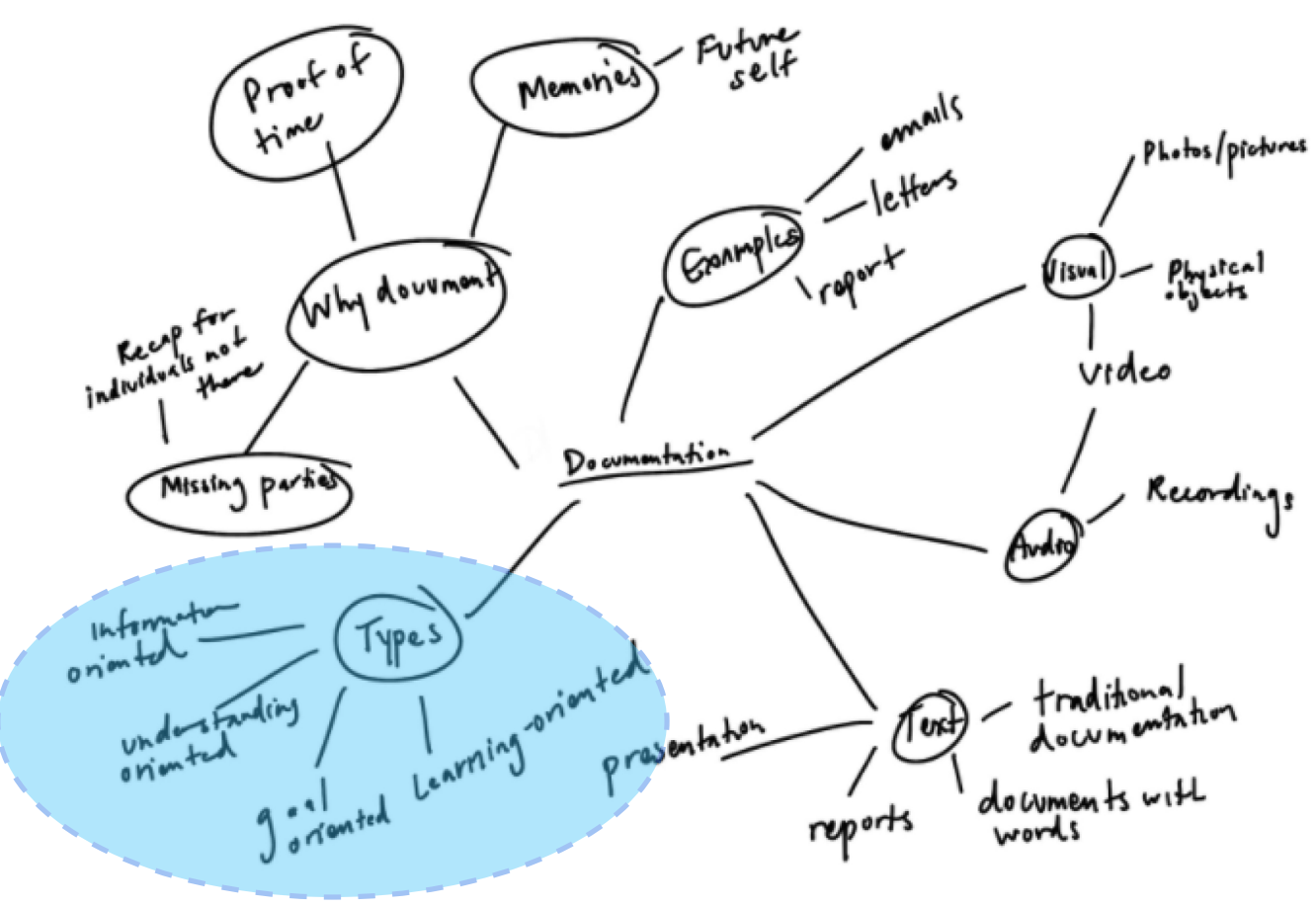

Participant 04 (Information Systems, Human-Computer Interactio

Participant 04 emphasizes the formats of documentation - textual, visual, audio - with examples. Documentation types are classified as information, understanding, goal, and learning-oriented. Again, memory and proof feature as nodes.

\section{PERSONAL MEANINGS CONTINUED}

The representative set of mind maps selected here shows that participants explained and related to the word 'documentation' quite differently. Student associations appear to be somewhat informed by their disciplinary perspectives, as products of their training and course requirements, as well as by personal reflections on the benefits and shortcomings from their own documentation practices. For example, P12 had a background in drama design and production and emphasised that project timeframes are critical for delivering performances to audiences. They noted documentation as crucial for aligning all project members' activities and understanding. They framed breakdowns in documentation as failure to meet due dates and highlighted documentation as supporting "survivability". Despite the rich conceptual variations portrayed in these maps, we also observed some areas of overlap in many of the mind maps. For example, we see terms such as proof, accountability, assessment, and evidence of effort. This language suggests that documentation may have a potential sense of oversight by peers, teammates, instructors, and even by oneself. Other terms such as memory/memories, revising past work, recording, survivability, and standing the test of time also feature in the maps, suggesting that an archival, keepsake quality comes with documenting one's work. Regarded all together, these mind maps attest to the richly conceived and important role documentation seems to play in creative practice learning domains. 


\section{VALUES \& APPRECIATION}

- - - - - - - _ - - - - In a subsequent activity, we further explored shared values and priorities by asking participants to list as many reasons as they can think of as to why they practice documentation. The participants were then asked to rate each stated reason on a scale of 1-5 (with 1 being the most valuable). Asking students to rank their reasons for documenting was intended to shed light on the motivations that lead students to document their work and the value of documentation activities.

We analyzed student responses using an iterative, bottom-up coding approach to identify emergent themes and ultimately aggregate the responses into twelve overarching categories. To visualize this data we preserved each given reason's assigned ranking (importance level) to a color gradient with the darkest color indicating most important and the lightest color meaning least important. This way we could see both the frequency of each of the twelve main reasons given for doing documentation by bar height and show the relative importance within each category by color saturation. In some cases, students reported multiple reasons in the same category. During coding, rankings were inverted ( 5 being the most valuable) to allow the scores to be aggregated.

\section{DOCUMENTATION BECAUSE.... \\ (5) SAUES ME TIME LATER \\ (4)HELPFUL FOR TEAM \\ - HEY CAN ENOU STATUS OF THE REST OF THE TEAM \\ - EATY FOR THEMTO FIND NOTES CIUEN IN MEETINC, PREVIOUS ITERATIONS \\ FUN TO LOOK BACK AT}

(2) CLEARGY DOCUMENTS PROCRESS + PROGR ESSION OF PROJECT

- MORE SASIIY RZPEATABLE

(3)- HELPFUL FOR POST MORTEM HEETINGS/REFLECTION

(1) Gathirs au information in a (ENTRAL hub (hopgFuly)

MAKSS LIEE EASIER DOWN TIE ROAD

- LESS tIME SPENT LOOKINL fOR PIECES OF INFORMATION

\section{REALIM LIKE MAKINK PAPERWORK}

\section{FEEIS LIKE THE RICHT THING TO DO}

\section{KEEP TRACK OF WFORMATION MORE SASILY}

+ EFFICIENTIY

$+\varepsilon F F \varepsilon c T$ IULCy
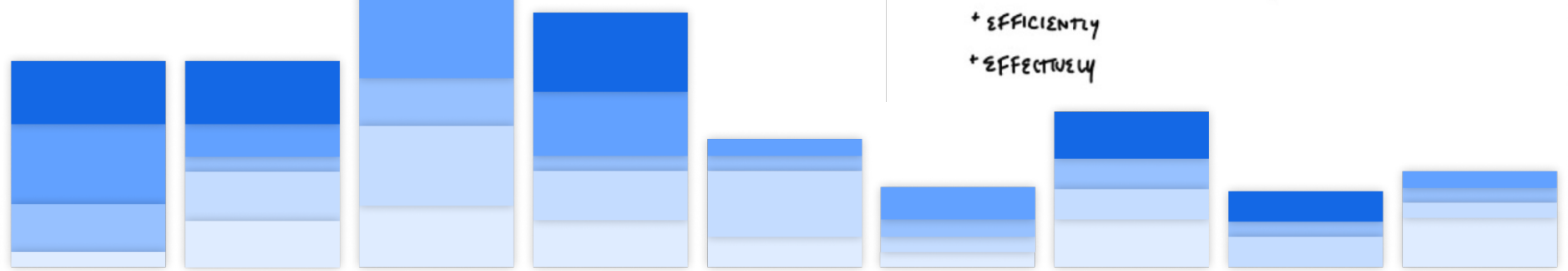

\section{Color Key}

Highest Priority

7 $\begin{array}{ccc}\mathbf{4} & \mathbf{5} & \mathbf{6} \\ \text { Communication } & \text { Metacognitive } & \text { Checkpoint }\end{array}$ Learning
8

Professionalism

\section{9}

Storytelling
10

Headspace
Evidence

\section{$6 \mathbf{I}$ I guess that it all makes me more thorough, in terms of the actual cognition and} thinking like how I came up with these ideas.

$4 \mathrm{I}$ It was not required by our instructor, but looking back, I think it would have been helpful to record the reasons behind our decisions... When we are discussing the decisions we are so focused on the current goal, but like, over time, maybe the situation might change.

\section{$6 \mathrm{For}$ me I think like the main driver for documentation is that it's just a process to} help me and my teams think.
From this analysis, we determined the five main reasons for documenting that were both shared and valued across disciplines. Notably, documentation as proof (or as a form of evidence) reemerged as a priority for students and was listed by 10 of 18 participants (with an aggregate score of 72). Similarly, students valued documentation's ability to support multiple forms of communication (86): coordinating within collaborative teams (33), sharing progress with others (21), and presenting ideas for feedback (32). Twelve participants noted that documentation supported metacognitive processes (62), including reflecting on experiences/mistakes, and helping to structure and make thinking visible. Participants also saw the benefits of documentation in tracing processes and keeping a record of past versions of work (68). In particular, documentation was viewed as a helpful inspirational resource for future work, in understanding how to trace the story of a project, fixing and learning from mistakes, and in tracking progress. Finally, documentation as a memory aid was recognized by 10 participants (47), and noted as a helpful way to recall the status of projects and to avoid forgetting processes and skills in the short and long term. 


\section{TRACING THROUGH THE DOCUMENTATION JOURNEY} For our third probe activity, participants prepared a journey map to represent their workflows and activities in a recent project, from beginning to end. Once completed, students were asked to annotate where documentation played a role in that journey, and mark challenges or missed opportunities encountered in this process. Selected projects were a mix of independent and collaborative from coursework. With the exception of one extracurricular project, projects were selected from coursework, representing a mix of independent and collaborative work. While the courses varied across contexts, pedagogies, and methods, the maps show that instructors and educators were formative in students' understanding of documentation, as necessitated through course requirements. The explicit and implicit expectations of course assignments and activities calibrated and shaped their subsequent practices. The journey maps revealed that many students enacted documentation using prescribed process terms with discrete stages, like user testing or between assignment milestones, while also working in iterative "loops", going back and forth between stages, from research to user interviews to back to research, for example. Our participants described being "trained" to use and apply specific processes and to implement established disciplinary "best practices".

The academic experience of managing work under rapid deadlines and piling demands, as well as pivots, evolutions, and constant change, was clearly evidenced with depictions of timelines, sequence, and iteration cycles. Nonetheless, the chronological convention of journey maps fostered linear arrangements of documentation. The disorder of the design or making process had to be ordered to be communicable. Instructions and deliverables necessitated certain forms of project presentation, involving students chunking their project progress into defined stages. Class deliverables like final papers or required process books had to cover the entire timeline of a project, which instilled anticipatory habits, like notes and photo-taking. Documentation being a required aspect of assignments also contributed to the sentiment that documenting was making for others - doing it for your professor and, because of remote learning, doing it for your peers too. These external demands seem to drive documentation needs.

An open question for us is to what degree do these documentation practices carry on into professional practice in creative fields once the academic requirements of documentation processes are removed?

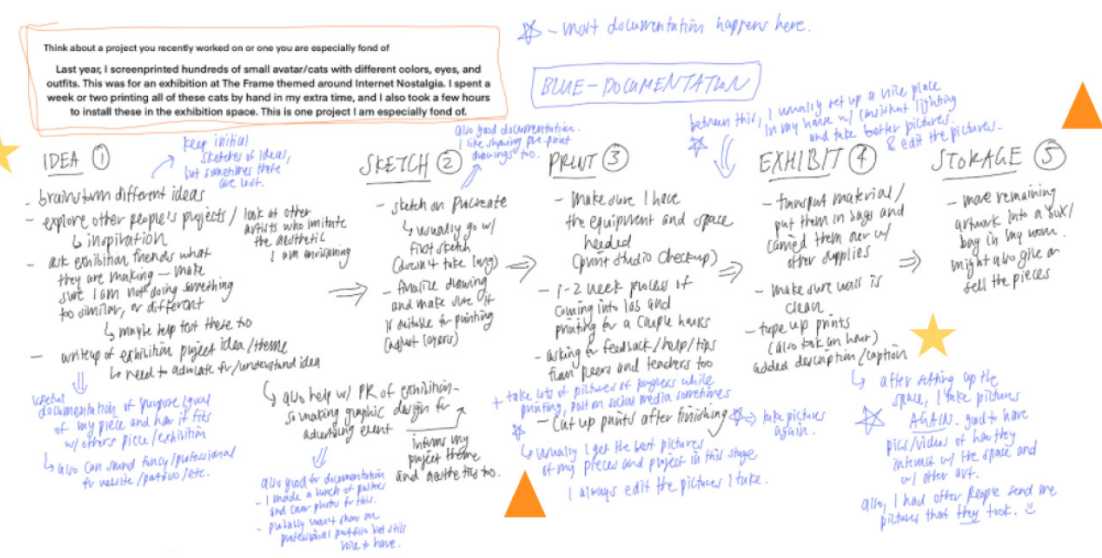

Participant 09 (Art, Psychology, Human-Computer Interaction)
Project Description: Website Redesign fora Professional Development organization our yolo was to intruiew users, ckated a new proposed design, and pautially implement the website (fiontend/tirmi/css).

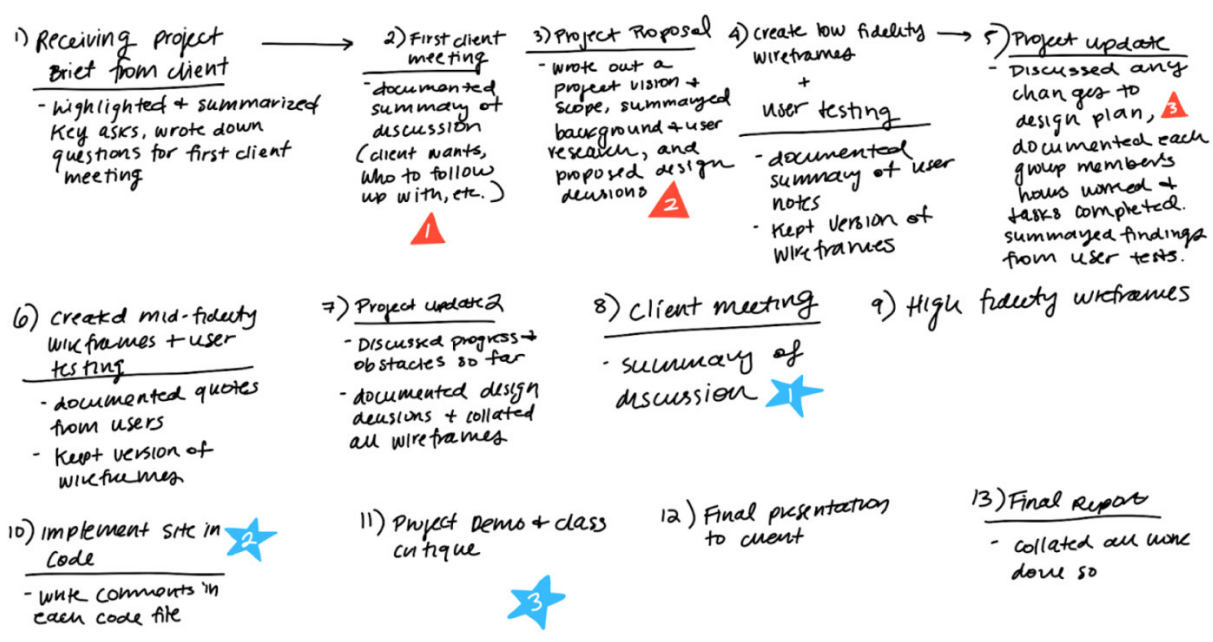

Participant 09 (Cognitive Science, Human-Computer Interaction, Media Design)

C $C$ I recently completed a semester-long project, in which documentation was built into our deliverables. By coming together as a group and documenting/summarizing our projects in intervals, it helped us periodically reassess our goals, take a look at our progress, and adjust our methods going forward. In this case, documentation for this big project was essential to make sure we stayed on track and it helped us not lose sight of our end goal.

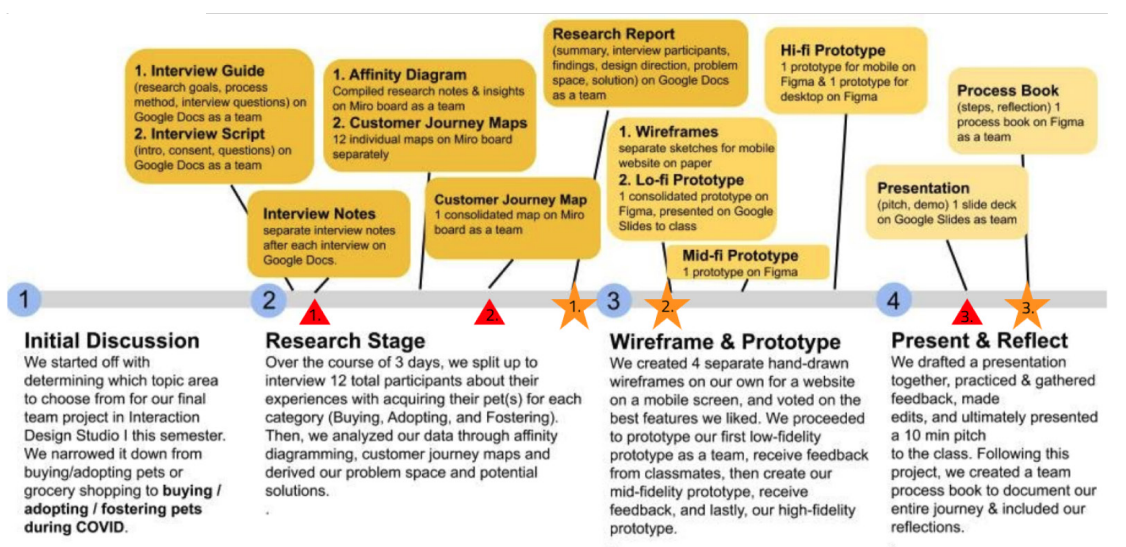

Participant 08 (Economics, Human-Computer Interaction) 


\section{ROLES OF TOOLS \& SPACE} The physical affordances of studio-type learning environments are important contributors to a shared culture and practice of documentation [5]. However, students during this time were working from non-school settings and did not have access to their regular tools and workspaces. The probe's first activity invited students to reflect on this by capturing a photo of their remote, often at-home, workspaces and to do so with their documentation practices in mind. Students were asked to consider their personal meanings of "documentation" as captured in their mind maps, and to annotate their photos to provide context on where things were situated and call out their roles in supporting their documentation practices. This activity was intended to reveal how students organize their workspaces to support creative project work and to highlight perceived challenges that resulted from no longer having access to the resources and creative supports found on campus. The annotated workspace photos provided visual inventories of students' equipments, tools, arrangements, and uses. Unsurprisingly, most students highlighted their laptop, tablet, or other digital devices as a primary focus of their workspaces, as the majority of work had transitioned to digital platforms.

In contrast, students with a dedicated work area were able to arrange valued resources to be ready-at-hand. While their at-home setups were considered to be "a lot different, lot smaller, than [their studio] desk[s]", these students recreated aspects of their studio to aid their work and documentation practices, with cutting mats, journals, sketch paper, and pin-up boards for keeping track of projects or placing inspirational resources.

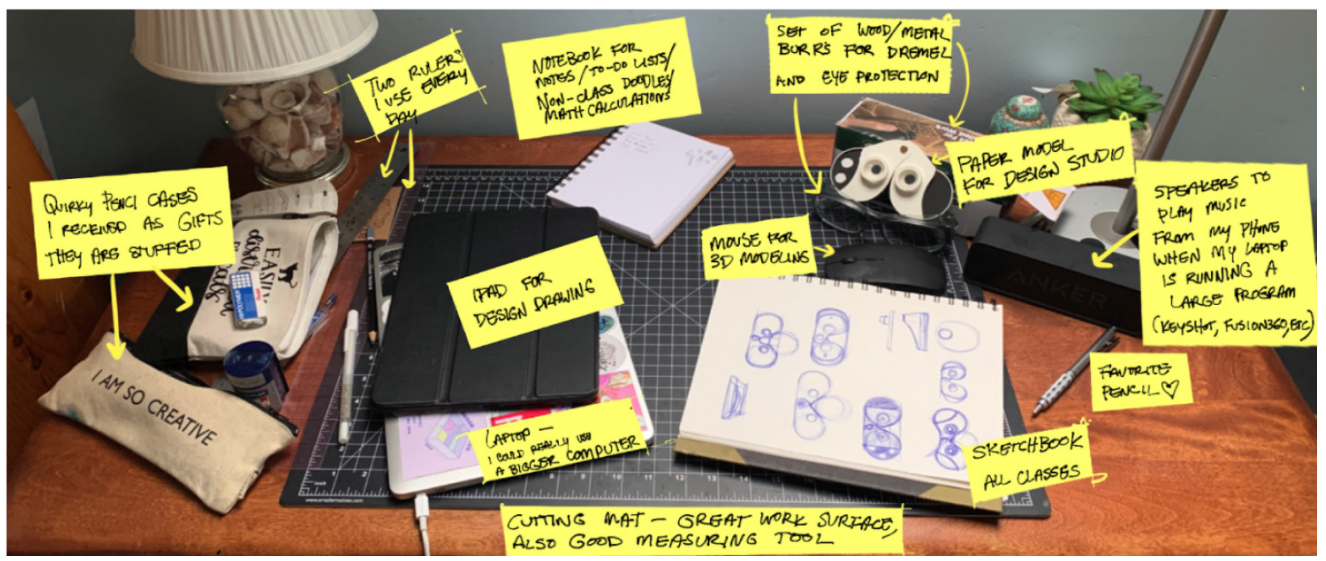

Participant 14 (Design, Physical Computing)

There were also many challenges that students experienced when working from home. This was particularly true of the four participants who relied on ad-hoc workspaces - repurposing beds, couches, and shared tables as places for work. This required agility, make-shift setups, and sometimes moving to new areas of the home to work throughout the day. This constrained the way in which environment and physical resources could support documentation practices.

$6 \mathrm{~T}$ This isn't my regular workspace. I like recently moved. I don't have a desk setup yet.
C6 I've learned to work at every single seat around the kitchen table because people also have to eat there and at home I have tried to bring my work down stairs more to spend more time with my family.

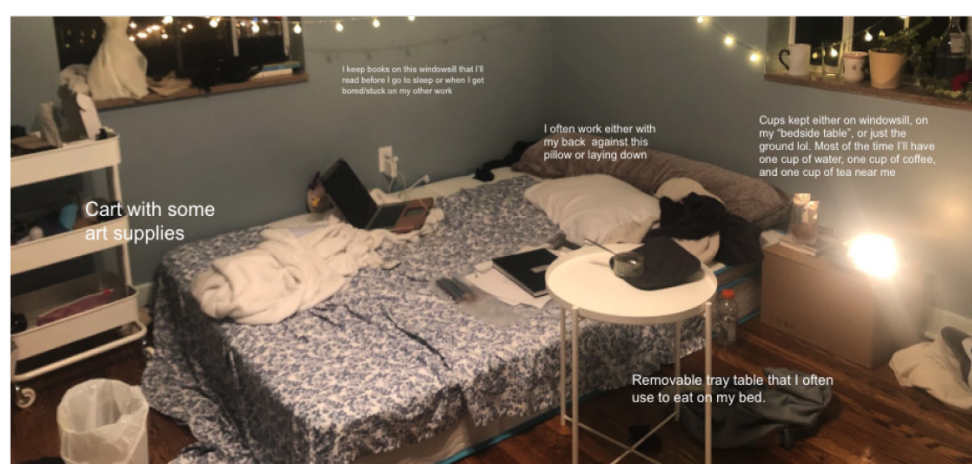

Participant 01 (Design, Human-Computer Interaction, Digital Humanities)
46 In the center I have what I'm using right now, my laptop and the book I'm using for my paper, and like off to the sides I have like my projects over here and like things I reached for on the other side.

64 For me, documentation is the process of gathering artefacts and tracking your thoughts, goals, and decision-making during the course of a project. I typically practice documentation in my workspace by physically writing things down. Often times this comes in the form of task lists on post-it notes or in a planner.
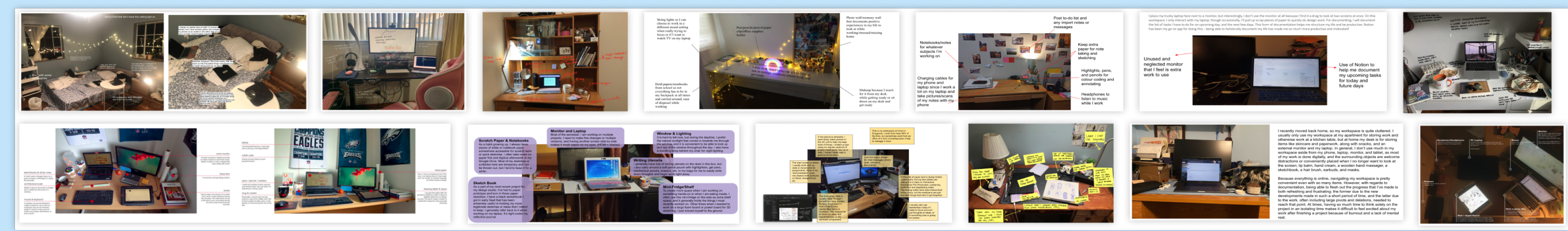


\section{DISCOVERING A LINGUA FRANCA}

the companison of probe activities, in combination with the refective interviews The comparison of probe activities, in combination with the reflective interviews with participants at the end of the week, allowed us to uncover shared experiences among students and commonalities found in their creative processes and documentation practices. From our analysis, we highlight a set of themes which seem

64 There was so many documents that came along the way we started out [..] So there was a bit of a sprawl and in the end, it was a little bit difficult to locate where things were. I don't know whether it's avoidable. It's a byproduct of how we're thinking, how we changing....suddenly we have a Google Doc every single week, or this file became extraordinarily large with everything from every single week.

The challenge of sprawl was a consistent theme we saw emerge out of the adoption-useabandonment cycle of various tools. In our interviews, this theme was often articulated as an effort to remember or recover something forgotten. Processes of digging back to locate items in time, in process, or in thought are in constant competition with managing the sprawl that necessitates navigating the physical and digital clutter accumulated. Passage of time and desired level of detail complicate efforts to revisit and refine documentation. For example, when the need arises during the process to find something (an idea, a decision, lines of reasoning, a quote, etc.), it demands urgency. The sprawl theme denotes this felt challenge of locating or recalling documentation to help with remembering, revising, demonstrating, or showing evidence of design activity.
$6 \mathrm{~T}$ Towards the end of the semester long projects I forget where we came from and we needed to start digging out things in order to make the process book...
C I kind of have to dig back to the time to locate where we were. So there were like different versions of documents.
$--------------------------------------$ to form a shared lingua franca that students use to communicate challenges and which we have characterized as sprawl, meta-level making, and the cartographer's dilemma.
C6 In the ideal world, you could write while making and that's what I know a few people do, but to me like it just feels very difficult and it's also quite frustrating to do so sometimes.

$4 \mathrm{Near}$ the beginning when I didn't really have an idea, I wrote, and then I made. But then near the end where I kind of like knew everything that was going on and I finally understood what was going on, it was more making and then writing.

Meta-Level Making refers to the added, secondary layer of making that process documentation requires on top of their project work. Documentation in the forms of captured snapshots, recordings, notebook sketches, or physical/digital mockups must be transformed into a polished visual narrative of one's thinking and making. In this step, the continuous, passive acts of capture become shaped and crafted into narrated outcomes. The sum of these captured (recorded) parts is less than a whole, because in their totality, these parts did not add up to meaningful documentation and so documentation was something more than just record keeping.
64 When I was thinking about it the most, it's also when I was writing about it the least ... I just wanted to make more and so I wrote less.
46 We often don't write that kind of meeting notes I think everybody's too involved in making the products.
Another salient theme that emerged in analysis is something that we coined as the "Cartographer's Dilemma," which characterizes the documentation challenge of knowing what features of the process to represent and which to elide. Students struggle to determine what level of visual and narrative resolution is needed to represent the details of the process and map the design terrain covered. The question arises as to what documentation is valuable? Students want to show - but not obscure with too much visual information, and tell—but without bogging down the construction of a storyline with too much extraneous detail.

These insights helped us formulate a preliminary hypothesis: students have motivations for and learning benefits from documentation that are shared, but pursued differently across disciplines. For example, preserving version history was important to 11 students, but this motivation meant understanding the factors that led to making some decision for one student, while for another it meant being able to follow the evolution of a form. Both students relied on having access to previous versions of their project and stages of their thinking to understand what to do in the present. Our work suggests students share a sort of lingua franca about documentation. We expect that a more explicitly formulated language around documentation could deepen learning processes and the connection between students and their creative inquiry, helping to extend the reflections from our probe into better established cultures of documentation. 


\section{PROBING AS A CATALYSIS FOR REFLECTION}

- - - - - - - - - - - - - - - - - - - - - - - - - - - - - - - - - - - - - Our probe package proved to be an apt tool for reflection by helping students draw together their various documentation practices, which serve purposes ranging from professional to instructional as well as personal. We examined how students' experiences speak to each other by focusing on the rich details of individual practices and reflections on personal experiences. In some cases we found that the probe activities catalyzed students to reexamine their assumptions:

4 Project documentation sounds like academic jargon a little bit.... I never really heard the term 'project documentation' before this, but I'm thinking about the activities like oh yeah I've done that before, I do that, kind of just instinctually. So it's good to, you know, kind of put a name on it. Like the idea of documentation is kind of just ingrained into what I'm used to doing for projects, so it was interesting to look back and examine the systems l've set up for myself.
66

I never really had given a thought to my practice of documentation and I don't honestly know, I didn't know the definitions of documentation until when I'm asked to do it, and then it really got me to think like oh, what exactly does it mean to document stuff and how exactly. I've been doing this to help myself. And then some of the probe questions you guys asked such as what I could have done to make the process better also helps me reflect on my experience because you just got me to think how l've been currently doing with my documentation practice and how I could have done it better to improve my experience, basically.
66

I usually don't think very critically of documentation, usually it just comes and it goes, more of an if it happens it happens kind of thing. It helps facilitate my thinking, helps drive our team forward, like maybe not always super useful, but after the fact it's written down .. it just helps us get stuff done .. figure out what we need to do next. I don't think any of it has been like a waste of time. Maybe some of the notes. It's just maybe less utilized by people.
For several participants documentation was simply an ingrained part of their design process that had never been explicitly labeled for them. In these cases, the retrospection facilitated by the probe activities played an active and meaningful role in uncovering students' learning processes for themselves. For some students, documentation was not something that had been explicitly facilitated as part of their studio experience or instruction. Students valued the probe as a reflexive tool to examine their own documentation and spoke to the educative value of the probe itself for personal sensemaking around documentation practices.
Together, the artefacts generated using the probe helped make visible practices and values. The final debrief conversation offered students a facilitated reflection on documentation and gave students an opportunity to articulate their experiences with the probe. These interviews helped to make sense of personal stances on documentation and to generate new interpretations of their practices.
While engagement with our probe helped students deepen their individual reflection on their experiences and valuing of documentation, there is much that this probe did not unpack or help students examine. Hinted at in the data are references to coordinating processes of teamwork and accountability, and the role documentation plays in mediating those interactions. Given how much of creative project work is collaborative, it would be a logical progression to prompt or encourage reflection among teams of students from varied backgrounds on how they are "speaking to" one another across disciplines through forms of documentation. This will be explored as part of future work. 


\section{CONCLUSION \& FUTURE WORKS}

In studio- and maker-based learning environments, documentation tools and collaboration platforms need to evolve with educator practices to facilitate and better support the learning routines and habits of mind that make thinking visible, share process and outcomes, and engage disciplinary-based critique of individual creative processes and learning. This week-long design probe investigated individual documentation practices among fifteen students from a range of creative domains. The probe activities made visible students' personal interpretations, appreciations, and practices related to documentation. We found that while the specific enactments through tools, technologies, and actions are not uniform across disciplines, students coherently recognized the important role documentation plays in their creative work, the learning benefits it offers, and the value of fostering a culture around this professional practice as part of their learning experiences.

Learning to flexibly and recursively model the trajectory of one's unfolding creative process is a form of design knowledge, and one which these probe activities scaffold [41]. The probe enabled students to draw together and attend to the details of their individual practices, as well as to the effects and influences of documentation habits on their creative inquiry. We believe that the probe offers a rich resource for reflection on personal and professional development. In future work, we intend to investigate this further by introducing the probe as an instructional tool within capstone studios. Additionally, it would be beneficial to deploy this probe across a greater diversity of students, learning contexts, and forms of creative practices, as well as to conduct further qualitative analysis of this rich dataset.

These activities, and the insight produced, will inform future work and the larger aim of this exploratory learning and design research project: to better understand how low-cost, tangible technologies can be used to support documentation practices in creative learning. We chose design probes as a generative method that gives voice to students' perspectives and that offered a methodologically grounded way to prefigure and reveal the less obvious aspects of documentation. Practical insights suggest how tangible tools can integrate with ad hoc, non-traditional workspaces. Broader opportunities in underlying motivations for documentation were also found in the probe outcomes. Inspired by the utility of the probe as a reflective tool, and by the lineage of work in scaffolding reflective practice through technology [22, 33], our future work will tend to designing technology that centers the artefacts of documentation in reflective practices.

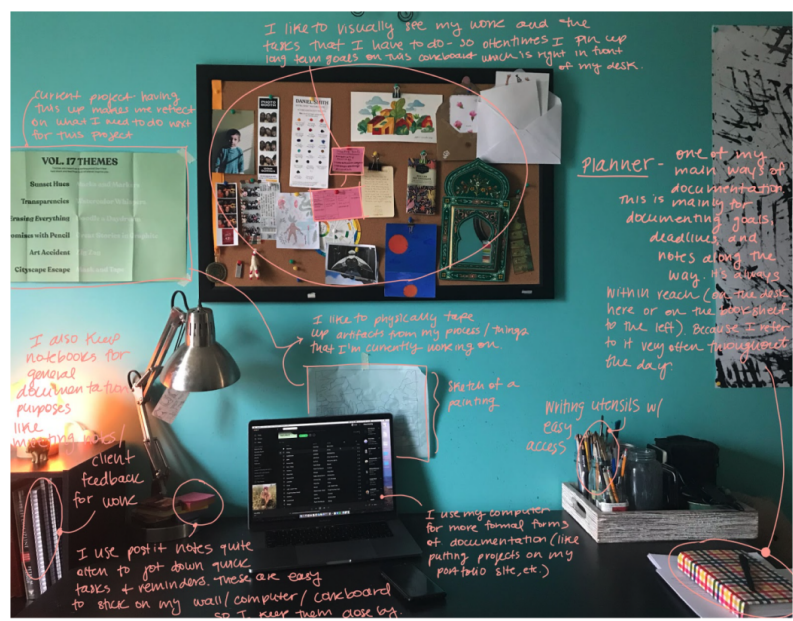

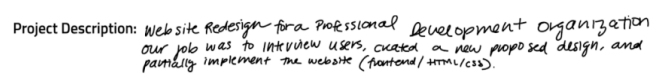

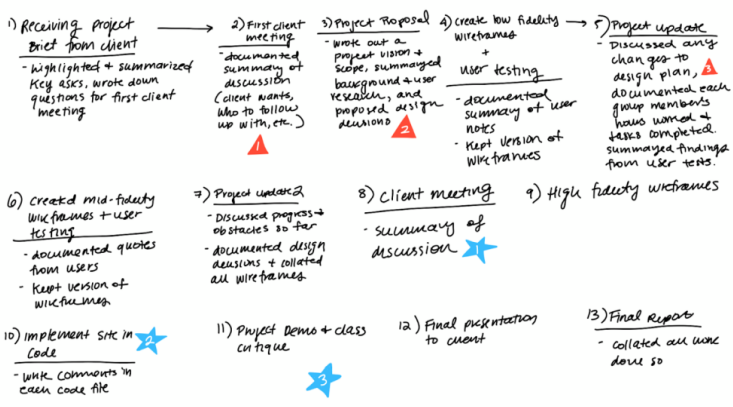

rintions

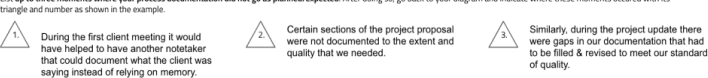

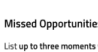

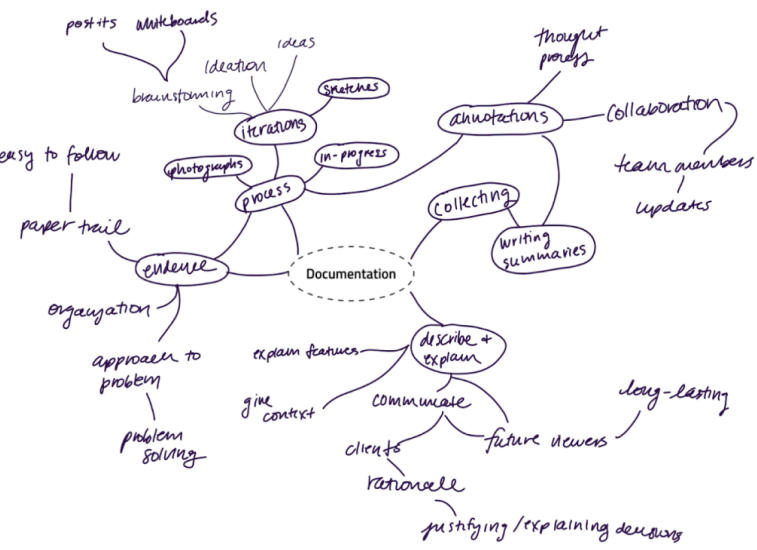

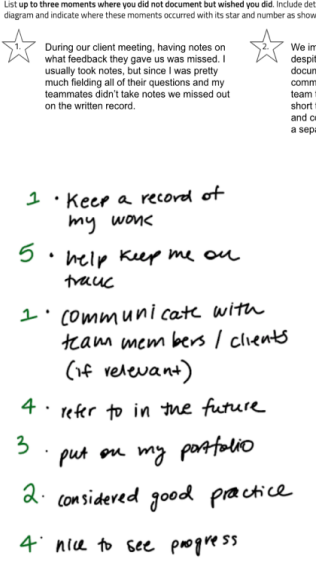

2. makes you be awore the deusions you make

2. justity uny you are taking a certem approach

3. good to kfer bave to order versions

5 . semes as a backecup

CC Documentation is not something I was familiar with until I started my $\mathrm{HCl}$ education as a lot of my experience with documentation started from design projects particularly through process book deliverables. While I mainly practice documentation as a tool of keeping record and facilitating communication with others, I still myself sometimes doing it due to societal pressure of saying it is considered a good practice.

C While remote-learning has disrupted a lot of the documentation habits that I am used to, it has also allowed me to adapt new habits where I focus on documentation as the key to improving communication and bringing my teammates and others on board faster.

All of the probe activity outcomes and two salient quotes from Participant 09 (Cognitive Science, Media Design, Human-Computer Interaction)

\section{ACKNOWLEDGEMENTS}

- - - - - - - - - - - - - - - - - - - - - - - - - - - - - - - -

We would like to thank our participants for their thoughtful engagement with the probe activity. This material is based upon work supported by the National Science Foundation under Grant No. 1736189 "Smart Spaces for Making: Networked Physical Tools to Support Process Documentation and Learning." 
[1] Brigid Barron and Linda Darling-Hammond. 2008. Teaching for meaningful learning: A review of research on inquiry-based and cooperative learning. Powerful learning: What we know about teaching for understanding 11 (2008), 70.

[2] Kirsten Berthold, Matthias Nückles, and Alexander Renkl. 2007. Do learning protocols support learning strategies and outcomes? The role of cognitive and metacognitive prompts. Learning and Instruction 17, 5 (2007), 564-577.

[3] Fiona Blaikie, Diederik Schönau, and John Steers. 2004. Preparing for portfolio assessment in art and design: A study of the opinions and experiences of exiting secondary school students in Canada, England and The Netherlands. International Journal of Art \& Design Education 23, 3 (2004), 302-315.

[4] Kirsten Boehner, Janet Vertesi, Phoebe Sengers, and Paul Dourish. 2007. How HCI Interprets the Probes. In Proceedings of the SIGCHI Conference on Human Factors in Computing Systems (San Jose, California, USA) (CHI '07). Association for Computing Machinery, New York, NY, USA, 1077-1086. https:// doi.org/10.1145/1240624.1240789

[5] Jana Bouwma-Gearhart, Yoon Ha Choi, Cindy A Lenhart, Idalis Villanueva, Louis S Nadelson, and Estefany Soto. 2021. Undergraduate students becoming engineers: The affordances of university-based makerspaces. Sustainability 13, 4 (2021), 1670.

[6] Robin Braun, Wayne Brookes, Roger Hadgraft, and Zenon Chaczko. 2019. Assessment design for studio-based learning. In Proceedings of the Twenty-First Australasian Computing Education Conference. 106-111.

[7] Judith O Brown. 2002. Know thyself: The impact of portfolio development on adult learning. Adult Education Quarterly 52, 3 (2002), 228-245.

[8] Tony Buzan and Barry Buzan. 2002. How to mind map. Thorsons London.

[9] S Chang, A Keune, K Peppler, A Maltese, C McKay, and L Regalla. 2015. Open portfolio project, phase 1: Research brief series.

[10] Edward P Clapp, Jessica Ross, Jennifer O Ryan, and Shari Tishman. 2016. Maker-centered learning: Empowering young people to shape their worlds. John Wiley \& Sons.

[11] Allan Collins and John Seely Brown. 1988. The computer as a tool for learning through reflection. In Learning issues for intelligent tutoring systems. Springer, 1-18.
[12] Allan Collins. 2006. Cognitive Apprenticeship. Cambridge Handbook of the Learning Sciences (2006), 47-60.

[13] Nigel Cross. 1982. Designerly ways of knowing. Design studies 3, 4 (1982), 221-227.

[14] Phillip Crowther. 2013. Understanding the signature pedagogy of the design studio and the opportunities for its technological enhancement. Journal of Learning Design 6, 3 (2013), 18-28.

[15] Andrew Danowitz. 2016. Instructables. com as a tool to improve student outcomes and promote community engagement (Work-in-progress). In American Society for Engineering Education Pacific Southwest Conference Proceedings. 330.

[16] Linda Darling-Hammond et al. 1993. Authentic Assessment in Practice: A Collection of Portfolios, Performance Tasks, Exhibitions, and Documentation. (1993).

[17] Martin Davies. 2011. Concept mapping, mind mapping and argument mapping: what are the differences and do they matter? Higher education 62, 3 (2011), 279-301.

[18] Kristen E DiCerbo. 2017. Building the evidentiary argument in game-based assessment. Journal of Applied Testing Technology 18, S1 (2017), 7-18.

[19] Hugh Dubberly. 2004. How do you design: A compendium of models. San Francisco, CA: Dubberly Design Office.

[20] Thomas A Dutton. 1987. Design and studio pedagogy. Journal of architectural education 41, 1 (1987), 16-25.

[21] Rowanne Fleck and Geraldine Fitzpatrick. 2009. Teachers' and tutors' social reflection around SenseCam images. International Journal of Human-Computer Studies 67, 12 (2009), 1024-1036.

[22] Rowanne Fleck and Geraldine Fitzpatrick. 2010. Reflecting on Reflection: Framing a Design Landscape. In Proceedings of the 22nd Conference of the Computer-Human Interaction Special Interest Group of Australia on Computer-Human Interaction (Brisbane, Australia) (OZCHI '10). Association for Computing Machinery, New York, NY, USA, 216-223. https:// doi.org/10.1145/1952222.1952269

[23] Howard Gardner. 1989. Zero-based arts education: An introduction to ARTS PROPEL. Studies in Art Education 30, 2 (1989), 71-83.

[24] William W. Gaver, Andrew Boucher, Sarah Pennington, and Brendan Walker. 2004. Cultural Probes and the Value of Uncertainty. Interactions 11, 5 (Sept. 2004), 53-56.
[25] Andrew S Gibbons, Elizabeth Boling, and Kennon M Smith. 2014. Instructional design models. In Handbook of research on educational communications and technology. Springer, 607-615.

[26] Heidi Given, Lisa Kuh, Debbie LeeKeenan, Ben Mardell, Susan Redditt, and Susan Twombly. 2009. Changing school culture: Using documentation to support collaborative inquiry. Theory into practice 49, 1 (2009), 36-46.

[27] Connor Graham, Mark Rouncefield, Martin Gibbs, Frank Vetere, and Keith Cheverst. 2007. How Probes Work. In Proceedings of the 19th Australasian Conference on Computer-Human Interaction: Entertaining User Interfaces (Adelaide, Australia) (OZCHI '07). Association for Computing Machinery, New York, NY, USA, 29-37. https://doi.org/10.1145/1324892.1324899

[28] Jeffrey A Greene, Rebekah Freed, and R Keith Sawyer. 2019. Fostering creative performance in art and design education via self-regulated learning. Instructional Science 47, 2 (2019), 127-149.

[29] David Hay, Ian Kinchin, and Simon Lygo-Baker. 2008. Making learning visible: the role of concept mapping in higher education. Studies in higher education 33, 3 (2008), 295-311.

[30] Anna Keune and Kylie Peppler. 2017. Maker portfolios as learning and community-building tools inside and outside makerspaces. Philadelphia, PA: International Society of the Learning Sciences.

[31] Stacey Kuznetsov and Eric Paulos. 2010. Rise of the Expert Amateur: DIY Projects, Communities, and Cultures. In Proceedings of the 6th Nordic Conference on Human-Computer Interaction: Extending Boundaries (Reykjavik, Iceland) (NordiCHI '10). Association for Computing Machinery, New York, NY, USA, 295-304. https://doi.org/10.1145/1868914.1868950

[32] Gaea Leinhardt and Madeleine Gregg. 2002. Burning buses, burning crosses: Student teachers see civil rights. Learning conversations in museums (2002), 139-166.

[33] Xiaodong Lin, Cindy Hmelo, Charles K Kinzer, and Teresa J Secules. 1999. Designing technology to support reflection. Educational Technology Research and Development 47, 3 (1999), $43-62$.

[34] Tuuli Mattelmäki and Katja Battarbee. 2002. Empathy probes. In PDC. 266-271. 


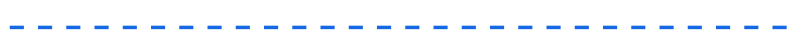

[47] Ron Ritchhart, Mark Church, and Karin Morrison. 2011. Making thinking visible: How to promote engagement, understanding, and independence for all learners. John Wiley \& Sons.

[48] Ron Ritchhart and David Perkins. 2008. Making thinking visible. Educational leadership 65, 5 (2008), 57.

[49] Elizabeth B-N Sanders and Pieter Jan Stappers. 2014. Probes, toolkits and prototypes: three approaches to making in codesigning. CoDesign 10, 1 (2014), 5-14.

[50] R Keith Sawyer. 2017. Teaching creativity in art and design studio classes: A systematic literature review. Educational research review 22 (2017), 99-113.

[51] R Keith Sawyer. 2018. Teaching and learning how to create in schools of art and design. Journal of the Learning Sciences 27, 1 (2018), 137-181.

[52] R Keith Sawyer. 2019. Dialogic status in design education: Authority and peer relations in studio class conversations. Social Psychology Quarterly 82, 4 (2019), 407-430.

[53] Donald A Schon. 1984. The reflective practitioner: How professionals think in action. Vol. 5126. Basic books.

[54] Leah Scolere. 2019. Brand yourself, design your future: Portfolio-building in the social media age. New Media \& Society 21, 9 (2019), 1891-1909.

[55] Alison Shreeve, Ellen Sims, and Paul Trowler. 2010. 'A kind of exchange': learning from art and design teaching. Higher Education Research \& Development 29, 2 (2010), 125-138.

[56] Cristen Torrey, David W McDonald, Bill N Schilit, and Sara Bly. 2007. How-To pages: Informal systems of expertise sharing. In ECSCW 2007. Springer, 391-410.

[57] John E Trowbridge and James H Wandersee. 2005. Theory-driven graphic organizers. In Teaching science for understanding. Elsevier, 95-131.

[58] Tiffany Tseng and Coram Bryant. 2013. Design, Reflect, Explore: Encouraging Children's Reflections with Mechanix. In CHI '13 Extended Abstracts on Human Factors in Computing Systems (Paris, France) (CHI EA '13). Association for Computing Machinery, New York, NY, USA, 619-624. https://doi. org $/ 10.1145 / 2468356.2468466$
[59] Tiffany Tseng, Maria Yang, and Stephen Ruthmann. 2014. Documentation in progress: Challenges with representing design process online. In International Design Engineering Technical Conferences and Computers and Information in Engineering Conference, Vol. 46346. American Society of Mechanical Engineers, VoO3T04A027.

[60] Belkis Uluo glu. 2000. Design knowledge communicated in studio critiques. Design Studies 21, 1 (2000), 33-58. [61] Yi LinWong and KinWai Michael Siu. 2012. A model of creative design process for fostering creativity of students in design education. International Journal of Technology and Design Education 22, 4 (2012), 437-450.

[45] Kylie Peppler, Anna Keune, Fangli Xia, and Stephanie Chang. 2017. Survey of assessment in makerspaces. Open Portfolio Project. Retrieve from https://makered.org/wp-conten in-Makerspaces. pdf (2017).

[46] Carlina Rinaldi. 2005. Documentation and assessment: What is the relationship. Beyond listening: Children's perspectives on early childhood services (2005), 17-28. 